\title{
Documents composites en didactique des sciences
}

L'usage de bandes dessinées

Composite documents in science didactics. The use of comic strips

\section{Catherine Goujon}

\section{CpenEdition}

Journals

Édition électronique

URL : http://journals.openedition.org/pratiques/8406

DOI : $10.4000 /$ pratiques.8406

ISSN : 2425-2042

Éditeur

Centre de recherche sur les médiations (CREM)

Référence électronique

Catherine Goujon, « Documents composites en didactique des sciences », Pratiques [En ligne],

185-186 | 2020, mis en ligne le 30 juin 2020, consulté le 15 octobre 2020. URL : http://

journals.openedition.org/pratiques/8406; DOI : https://doi.org/10.4000/pratiques.8406

Ce document a été généré automatiquement le 15 octobre 2020

(c) Tous droits réservés 


\section{Documents composites en didactique des sciences}

L'usage de bandes dessinées

Composite documents in science didactics. The use of comic strips

Catherine Goujon

\section{Introduction}

1 Notre article illustre des solutions d'utilisation de bandes dessinées (BD) dans nos écrits scientifiques en didactique des sciences. Nous explorons des extraits d'un corpus limité à nos propres écrits : notre mémoire de Master (Goujon, 2013), notre thèse (ibid., 2016), un poster présenté lors d'un colloque de didactique des sciences (ibid., 2018) et un chapitre d'ouvrage (ibid., 2019). Ils s'adressent à d'autres chercheurs ou à des professeurs (en poste ou en formation). Nos écrits abordent le thème de la didactisation des savoirs scientifiques, dans le cadre de la fête de la science, par des physiciens, hydrogéologues ou des biologistes, à un public de non-connaisseurs.

2 L'usage de photogrammes et de séquences de photogrammes de type BD se développe dans les écrits scientifiques en didactique (Roesslé, 2009 ; Morales Ibarra, 2014 ; Besnier, 2016). Aussi, nos productions présentent-elles des vignettes ou des successions de vignettes combinées à d'autres éléments textuels ou iconiques: elles ne sont ni exclusivement des BD ni seulement du texte. Elles sont un tressage des deux: des documents composites. Nous empruntons l'analogie du tressage à l'anthropologue T. Ingold (2017, p. 31) qui montre « comment une forme surgit d'un mouvement, mais aussi les propriétés dynamiques d'un matériau». Par ailleurs, nos productions comportent d'autres éléments mis en systèmes: plans, tableaux, graphes, frises chronologiques, schémas, etc. Nous ne nous intéressons ici qu'aux photogrammes traités en BD combinés au texte.

3 Nous proposons de caractériser notre méthode au regard de choix épistémologiques et théoriques qui trouvent leurs racines du côté de recherches en didactique mais aussi du 
côté de recherches spécifiques sur la bande dessinée. Nous situons nos travaux dans le cadre de la théorie de l'action conjointe en didactique (TACD). Nous nous nourrissons des travaux de G. Sensevy $(1999,2011 a, 2011 b, 2013)$ sur les méthodes pour rendre compte de l'action didactique, de ceux de D. Forest (2006) sur l'utilisation des photogrammes dans les études de proxémique et de ceux de J.-N. Blocher (2018) sur les systèmes hybrides texte-image-son (SHTIS). Par ailleurs, nos productions et les effets recherchés sont analysés avec l'éclairage des études de théoriciens de la bande dessinée. W. Eisner (2010 [1996]), le père de l'expression " art séquentiel », décrit et analyse la " narration visuelle" qu'il pratique lui-même en tant que dessinateur. S. McCloud (2007) et T. Groensteen (2008), dans son sillon, précisent la grammaire de cet art au regard de nombreuses œuvres internationales qu'ils étudient.

4 Nos vignettes et séquences de vignettes sont proches de celles de la $B D$ de reportage. Elles sont extraites de photogrammes, et les scénarios sont dictés par les événements et les résultats de nos enquêtes. Ce type de $\mathrm{BD}$, dite « de reportage », est un genre qui se répand. Dans l'album Le photographe, Guibert, Lefèvre et Lemercier (2010 [2003]) racontent une expédition humanitaire en Afghanistan. Ils y alternent des photographies sans recadrage et des dessins, à la manière de Hergé, réalisés sur le terrain ; dans l'album La Lune est blanche, les frères Lepage (2014), l'un photographe, l'autre dessinateur, racontent leur aventure lors d'une expédition scientifique en Antarctique. Les planches alternent là encore des dessins et des photographies; le journaliste C. Spottorno et le photographe G. Abril (2017 [2016]) ont produit un album, La fissure, composé de leurs photos prises lors d'un reportage sur la vie des migrants en Europe. Ces photographies sont filtrées en mode bande-dessinée pour limiter l'effet affectif tout en respectant les données empiriques récoltées.

Les documents que nous produisons reprennent ces éléments ethnographiques. Cependant, ils sont plongés dans et articulés à nos textes.

Concevoir et lire ce type de documents composites qui lient séquences de vignettes et texte demande un éclairage sur la production et lecture de ces espaces. Aussi, dans une première partie, nous expliciterons l'ancrage épistémologique et théorique de notre démarche. Nous préciserons ensuite la genèse des images que nous utilisons.

\section{Ancrage épistémologique}

7 Notre travail sur l'introduction de la bande dessinée dans nos écrits est une partie de notre réflexion sur des documents composites que nous nommons Systèmes Hybrides Texte-Image-Son (SHTIS). Ce sont des représentations composées d'éléments recueillis par le chercheur (ou l'ingénierie coopérative) dans le cadre d'une recherche, et d'éléments créés par le chercheur pour mieux rendre compte de la pratique. Ils sont mis en système pour établir les relations : un extrait vidéo, le verbatim associé, un plan $\mathrm{du}$ lieu de l'action, une frise chronologique, une description ou une analyse (textes)... Les plus riches sont multimodaux et hypertextuels. Leur ontologie est intrinsèquement liée à la volonté et à la nécessité donner à voir et à comprendre la pratique étudiée, et les indices qui conduisent le chercheur (l'ingénierie) à ses résultats.

La forme la plus générale d'un SHTIS s'établit autour d'un film d'étude de cette pratique. Ce film fait l'objet d'annotations mises en système. Parmi ces annotations figurent des commentaires (qui peuvent venir des participants à cette pratique, ou d'analystes externes de cette pratique), des récits, des systèmes graphiques 
d'identification de tel ou tel élément saillant dans la pratique, des systèmes de jeu d'échelle qui situent la pratique étudiée dans un ensemble ou qui montrent sa dynamique temporelle interne, etc. Chaque annotation peut être a priori mise en relation avec n'importe quelle autre. Le STHIS s'organise précisément autour de ces annotations réciproques entre ses différents éléments. Le SHTIS est donc à la fois un outil de description (le réel est "parlé » avec des éléments digitaux tels par exemple des mots et expression) et un outil de dépiction (le réel est "donné à voir » avec des éléments analogiques comme les images filmiques). (Collectif Didactique pour Enseigner, 2019, p. 604-605) pour lui-même (avant leur diffusion). Le chercheur construit un savoir à partir de ses propres données empiriques (concret d'un réel particulier dont le film d'étude est un analogon), et d'un voir-comme qui les oriente.

La TACD s'appuie sur une notion de l'institution proche de celle de Mary Douglas (en relation avec les conceptions d'Émile Durkheim), qui peut faire voir l'institution comme une machine à construire des catégories de perception, d'appréciation et d'action légitimes. Une institution se caractérise ainsi toujours comme une manière particulière de voir le monde. En TACD, on dira donc qu'une institution produit un voir-comme (dans le sens de Ludwig Wittgenstein). (ibid., p. 598)

Un SHTIS pourrait alors être comparé à un bureau virtuel où le chercheur, avec son voir-comme scientifique, rassemble différentes données. Ainsi se donne-t-il « les moyens conceptuels et méthodologiques de décrire et penser les processus de diffusion du savoir » (Sensevy, 2011b, p. 230).

\section{Du côté du lecteur}

La conception de SHTIS se joue dans leur production, mais aussi dans les choix opérés pour leur diffusion et leur réception. Nous considérons le SHTIS comme un milieu avec lequel le lecteur doit agir, avoir une transaction, pour accéder à un savoir nouveau. Dans la TACD, le milieu est l'ensemble des éléments (formes symboliques) dont « l'élève » doit s'emparer pour en comprendre le système. Par analogie, le document composite est un système dont le lecteur doit pouvoir s'emparer.

Nous faisons l'hypothèse qu'offrir au lecteur plusieurs "versions" d'une même situation en augmente la compréhension. Avec G. Sensevy (2011a, p. 222-223), nous empruntons à N. Goodman (2011 [1968]) les concepts de description et de dépiction. La description désigne ici le système symbolique digital du texte. La dépiction correspond aux représentations analogiques (dans ce cas, les photogrammes ou séquences de photogrammes) plus denses que le texte car susceptibles d'engendrer différentes descriptions, associées à différentes interprétations. 


\section{Conséquence sur la présence des photogrammes traités telles des vignettes de $\mathrm{BD}$}

13 L'analyse quantitative des éléments graphiques présents dans notre thèse de doctorat (Goujon, 2016) révèle que la majorité des pages composites intégrant des images de style BD se trouvent dans les chapitres dédiés aux études de cas, dans leurs parties descriptives et analytiques (Figure 1). Nous y trouvons, en moyenne, deux photogrammes ou séquences de photogrammes (traités graphiquement ou pas) par double page. Dans la BD, comme dans nos écrits, la double page est celle qui s'offre à la vue du lecteur. Elle est organisée comme un champ panoptique (Bentham, 2002 [1791]) qui permet d'embrasser différents éléments combinés qui ont trait à une même situation.

Figure 1 : Répartition des éléments graphiques dans les différents chapitres de notre thèse en didactique des sciences (Goujon, 2016)

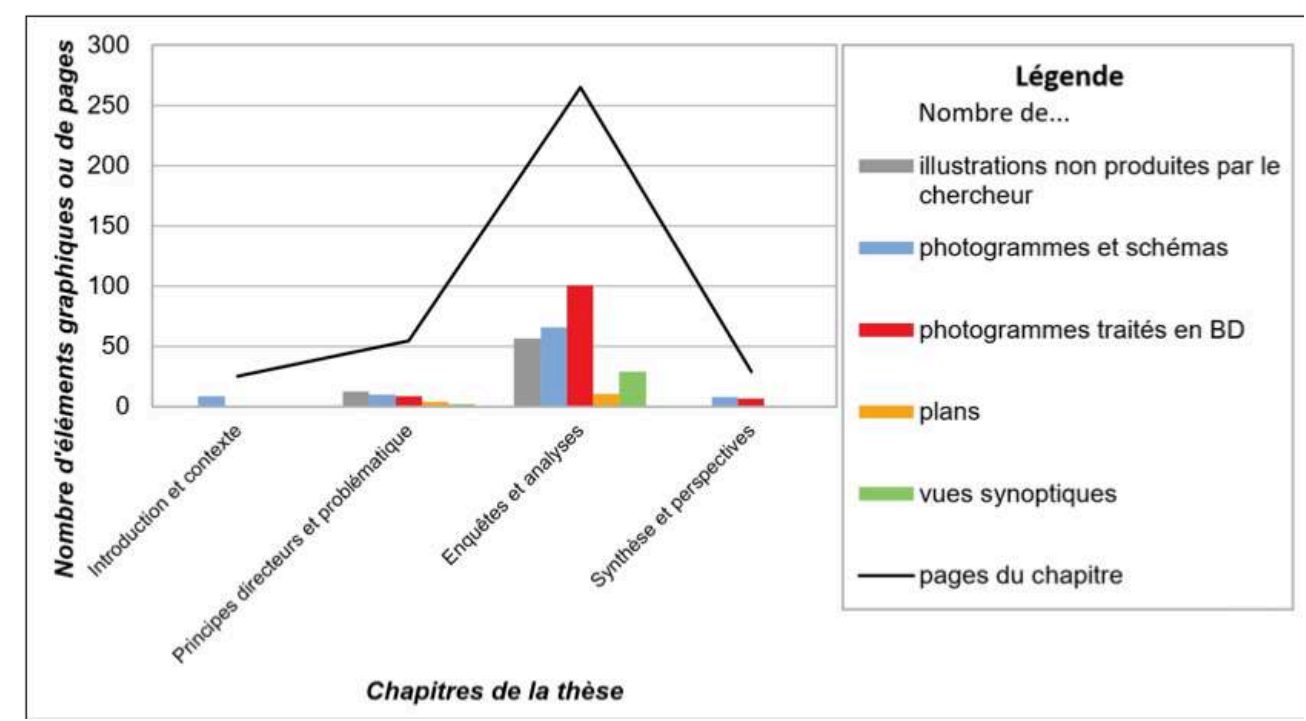

\section{Genèse des images}

\section{Contexte}

14 L'objet de nos travaux repose sur les pratiques effectives d'enseignement/ apprentissage. Les situations abordées ci-dessous concernent des animations scientifiques à la fête de la science ${ }^{2}$ («Le sable dans tous ses états "; "Os et articulations »; "L'eau du robinet, ça coule de source?») et une visite dans le laboratoire de recherche en physique des granulaires d'où sont issus les scientifiques médiateurs du stand sur le sable.

\section{Du réel au film d'étude puis au photogramme}

Les films d'étude que nous créons sont les données premières de nos recherches. Ils permettent de voir ce qui n'est pas immédiatement perçu. Ce sont des «systèmes d'inscriptions qui se substituent aux phénomènes que l'on veut étudier » (Sensevy, 
2011a, p. 218). Ils restreignent le champ des observables (d'où la multiplication des caméras pour augmenter les points de vue ou l'utilisation de caméras à $360^{\circ}$ et la présence souhaitée du chercheur sur le site), mais amplifient nos possibilités de perception, d'analyse et de témoignage : relecture, ralenti, accélération, pause, zoom, calcul de durées, extractions, sous-titrage...

Les images que nous publions sont le plus souvent des dérivées de photogrammes (images extraites des films d'étude). Nous figeons des scènes à des moments que nous jugeons décisifs dans le flux de la vidéo. Nous sélectionnons ces instantanés, en tant que connaisseur des pratiques des situations décrites, et les analysons. Nos opérons nos choix en fonction de l'objet de nos communications et donnons, si possible, au lecteur l'accès à la vidéo (voir le $\mathrm{QR}$ code et le lien sur le poster, Figure 2).

\section{De l'image photographique à l'image graphique}

17 Le filtrage du film avec un effet BD vise à orienter le regard du lecteur sur ce qui fait l'objet de la description ou de l'analyse de l'auteur-chercheur, par exemple, le contexte ou la gestuelle (Figure 2), des éléments de proxémique (Hall, 1971 [1966]). La technique graphique du silhouettage (Forest, 2006 ; Winkin, 2001 [1996], p. 166-185) a un lien de parenté avec la ligne claire ${ }^{3}$ utilisée en BD. Pour cela, un filtre " cartoon ${ }^{4}$ " est appliqué sur la vidéo. Cette technique améliore la lisibilité des images ${ }^{5}$ et nous obtenons ainsi une unité graphique pour tous les photogrammes d'une même situation.

Figure 2 : Document composite avec photogrammes silhouettés - Extrait du poster (Goujon, 2018)

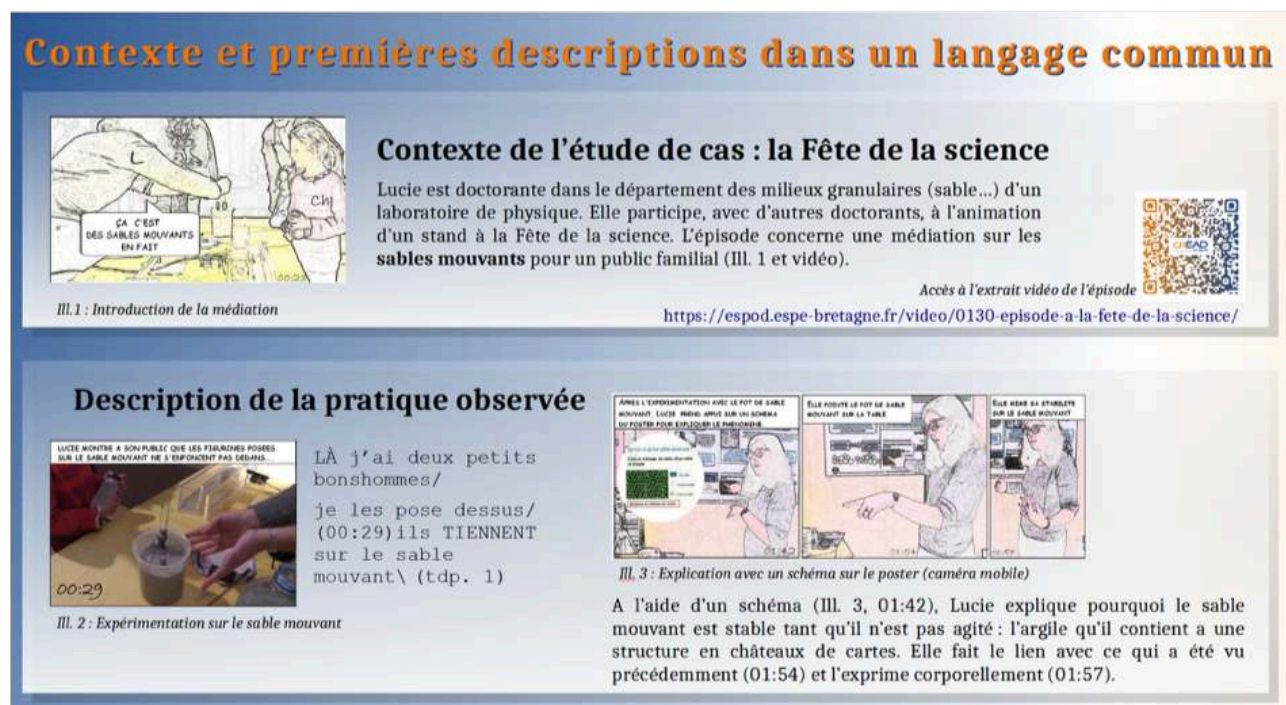

Les films d'où sont extraits les photogrammes, ainsi filtrés, conservent la nature analogique de l'image avec la situation. L'effacement des traits de leur visage produit une prise de distance par rapport aux personnes réelles. Ce choix technique est ancré dans une épistémologie. L'image agit comme une synecdoque: les personnes représentées ne sont plus seulement des individus. Elles représentent des instances : un professeur $(\mathrm{P})^{6}$, ici physicienne-médiatrice à la fête de la science, en face de son public considéré comme ses élèves (Figure 2) ; un scientifique présentant son travail ordinaire, un professeur (P), à la visiteuse de son laboratoire de physique (Figure 3), son élève (E). 


\section{forme photographique.}

Figure 3 : Instances représentées dans les vignettes - Extrait du chapitre d'ouvrage (Goujon, 2019)

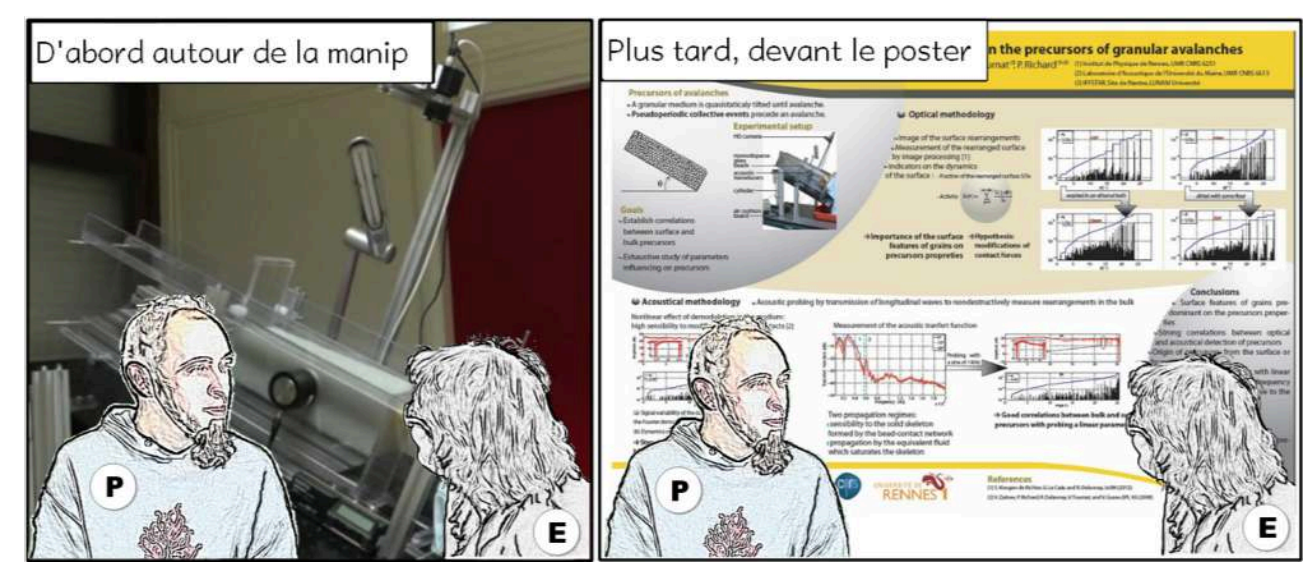

\section{Autres traitements de l'image fixe}

La réduction du corpus est suivie d'autres traitements des images. Un recadrage sur le sujet, un estompage de l'environnement, et une éventuelle retouche des silhouettes permettent de rendre la situation plus intelligible et lisible sur son support d'édition (Figure 4 et Figure 5).

Figure 4 : Photogramme sans filtre - Extrait du corpus de la thèse (Goujon, 2016)

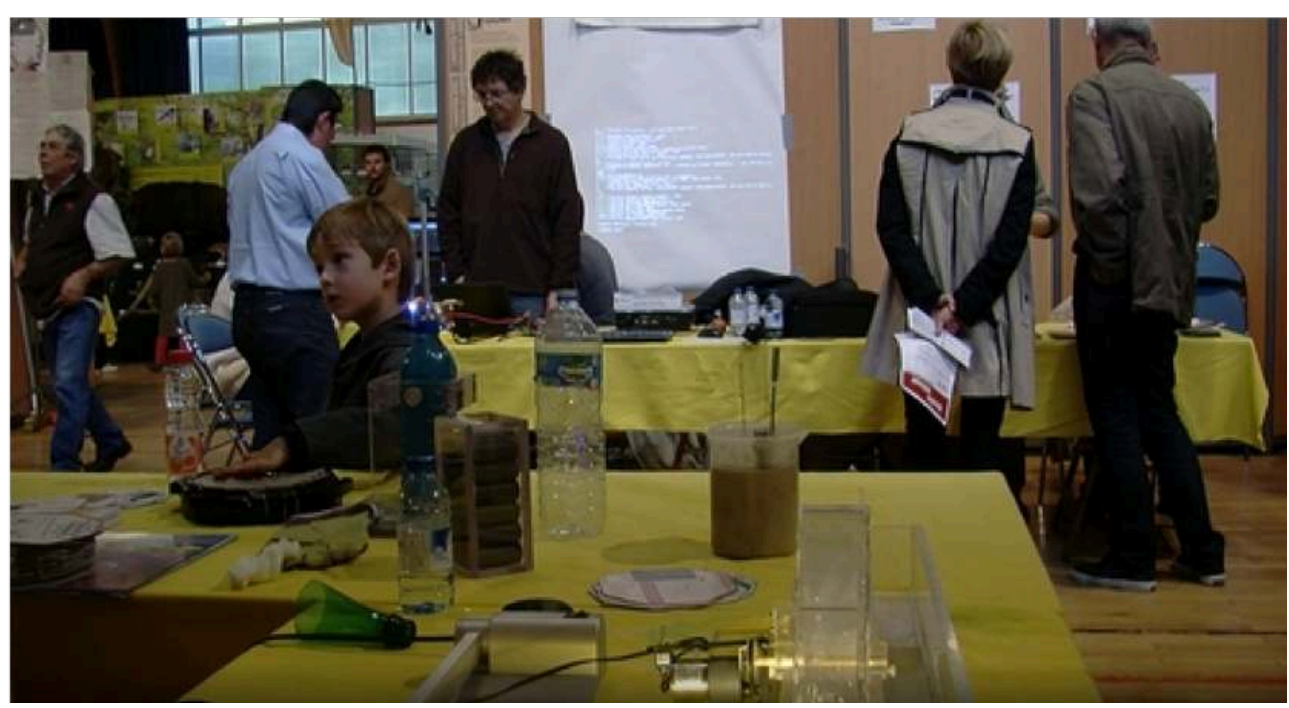

En effet, contrairement à la production de bandes dessinées, d'œuvres picturales ou photographiques, les scènes que nous filmons ne sont pas totalement scénarisées à l'avance. L'histoire est décryptée, décrite et analysée à posteriori. La position de la caméra est étudiée, mais les acteurs agissent à leur guise et sortent parfois du champ ; nous ne maîtrisons pas l'éclairage, les teintes de l'environnement ni les vêtements. 
Figure 5 : Photogramme extrait de la vidéo filtrée avec des effets, puis traité et annoté - Extrait de la thèse (p. 342)

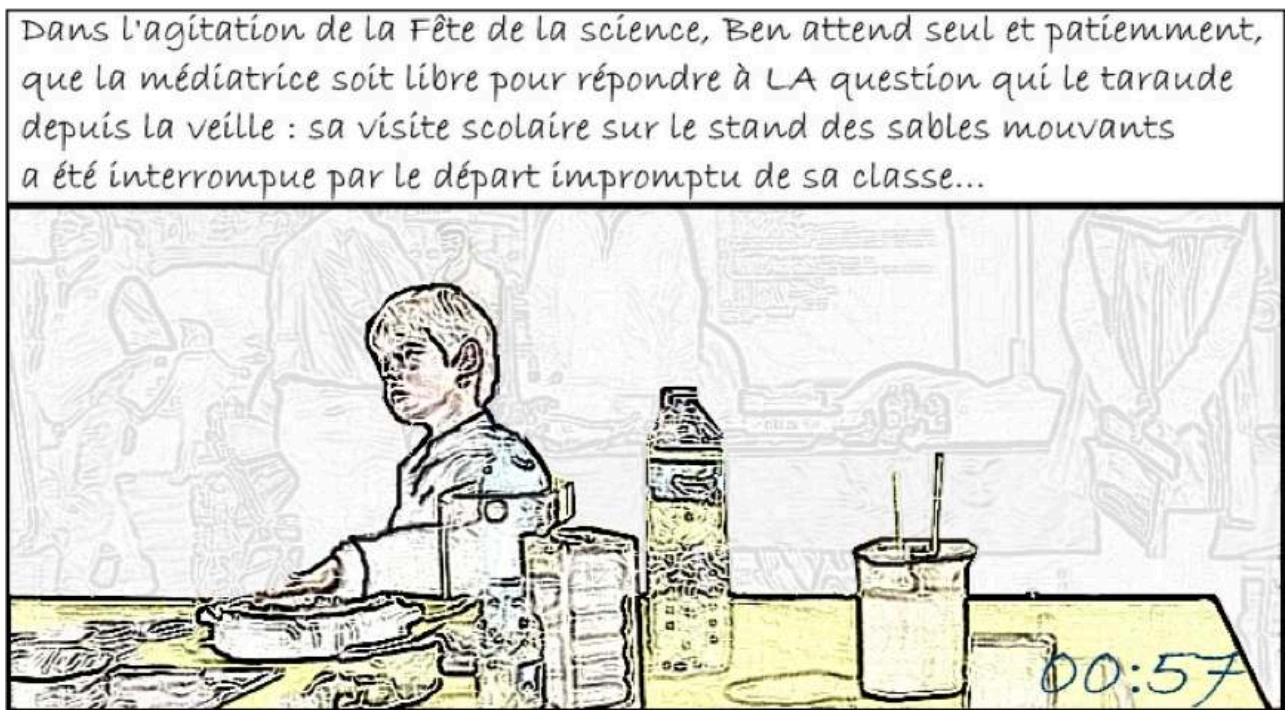

\section{Une rhétorique graphique empruntée à la bande dessinée}

Même si nos productions scientifiques sont essentiellement textuelles, les procédés de narration par l'image sont comparables à ceux de la BD. Nous décrivons ci-dessous quelques solutions graphiques d'ordre rhétorique.

\section{Séquence d'images et espace intericonique}

Plusieurs types de séquences d'images sont utilisés.

\section{« De moment à moment »}

La décomposition cinématique d'une action est un héritage des expériences chronophotographiques de E. Muybridge (1878). Antérieure au cinéma, la technique photographique de E. Muybridge permet d'analyser les mouvements. Une même action est représentée par une séquence d'images fixes avec un effet de continuum, "de moment à moment » (McCloud, 2007 [1999], p. 78). La lecture intericonique y est aisée. Le lecteur recrée intérieurement le mouvement. Les dessinateurs de BD utilisent ce procédé de narration. Dans Les récrés du petit Nicolas (Goscinny et Sempé, 1961), Sempé développe l'aveu du personnage «Papa, j'ai cassé le vase rose du salon» dans une séquence dessinée qui fait revivre la scène et l'angoisse de l'enfant. Le lecteur y suit, comme au ralenti, la trajectoire du ballon du Petit Nicolas. Le projectile bouscule le vase qui branle et tombe en miettes sur le sol.

Des physiciens, même, dans des revues scientifiques (Figure 6), se sont emparés de ce procédé. 
Figure 6 : Décomposition cinématique : enfoncement d'un objet dans un sable mouvant agité (Khaldoun et al., 2006)
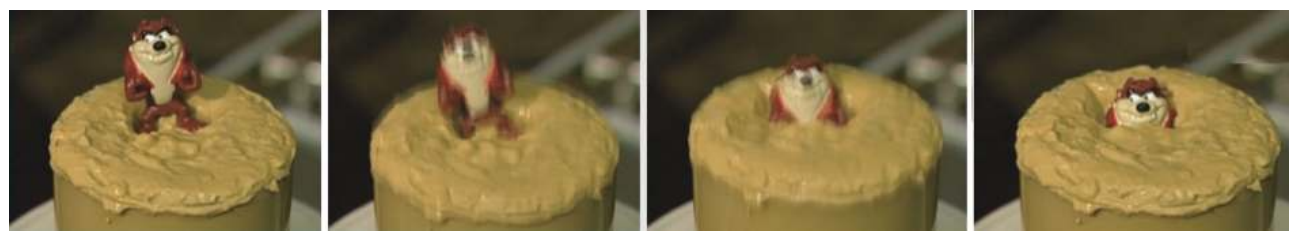

Nous l'utilisons pour la dépiction d'une seule action dont la description ne demande pas de découpage, même si la décomposition du mouvement peut être analysée.

Figure 7 : Séquence de photogrammes présentée en bande - Extrait de la thèse (Goujon, 2016, p. 314)

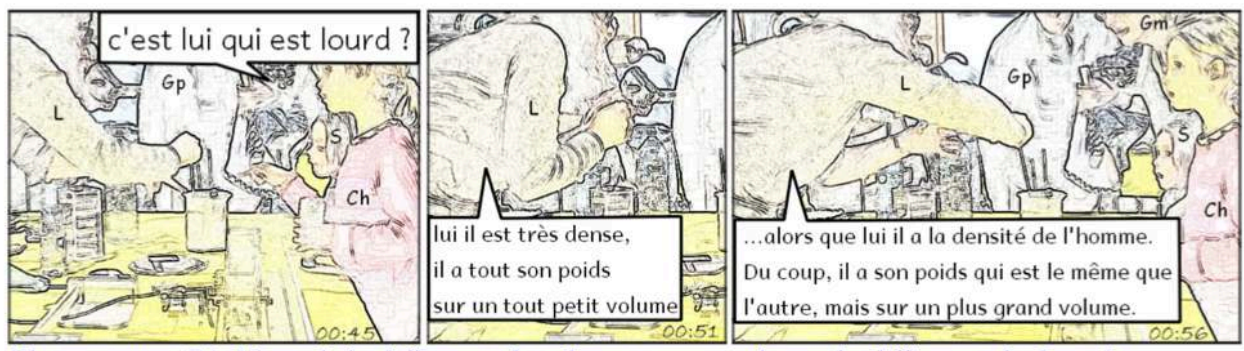

Photogramme 76: Mime de la différence de volumes, pour expliquer la différence de densités

L : lui il est très dense c'est-à-dire qu'il a tout son poids sur un tout petit volume ((mains et coudes serrés)) alors que lui il a la densité de $l^{\prime}$ homme du coup il a son poids qui est le même que l'autre mais sur un plus grand volumel (mains et coudes écartés)) (tdp. 3)

La bande de trois vignettes (Figure 7) illustre comment Lucie, la médiatrice scientifique, exprime la notion de changement de volume. Son action peut se décomposer ainsi : elle ressert les bras contre son corps (entre les vignettes 1 et 2), puis leur donne plus d'amplitude (entre les vignettes 2 et 3 ). Une légende unique décrit la séquence doublée de la transcription du discours de la médiatrice. Notons que la traduction analogique (photogrammes) donne beaucoup plus d'informations contextuelles que la transcription digitale du seul discours.

\section{« D'action à action »}

En revanche, certaines séquences d'actions doivent être décrites image par image, " d'action à action » (ibid.). Les sujets, les cadrages et le traitement des images ne sont pas forcément les mêmes (voir le contre-champ entre les illustrations 1 et 3 de l'extrait de poster, Figure 2, et la vue en plongée de l'illustration 2). Ce processus phasé peut être décrit par « et puis... et puis... ». Dans la BD, le lecteur doit créer les inférences pour comprendre ce qu'il se passe dans l'espace intericonique. Cet effort de déchiffrement des ellipses fait partie du plaisir littéraire. Mais dans un cadre scientifique, les informations sont univoques et précises pour limiter les erreurs d'interprétations. Selon les types de production de l'auteur scientifique, les solutions pour combler ce vide de l'espace intericonique diffèrent. La séquence des mêmes photogrammes, présentée sous la forme d'une bande avec didascalies sur le poster (Figure 8), est tressée au récit et à la description de la situation dans le cas de la thèse (Figure 9, p. 315). 
Figure 8 : Bande de trois vignettes commentées oralement - Extrait du poster (Goujon, 2018)

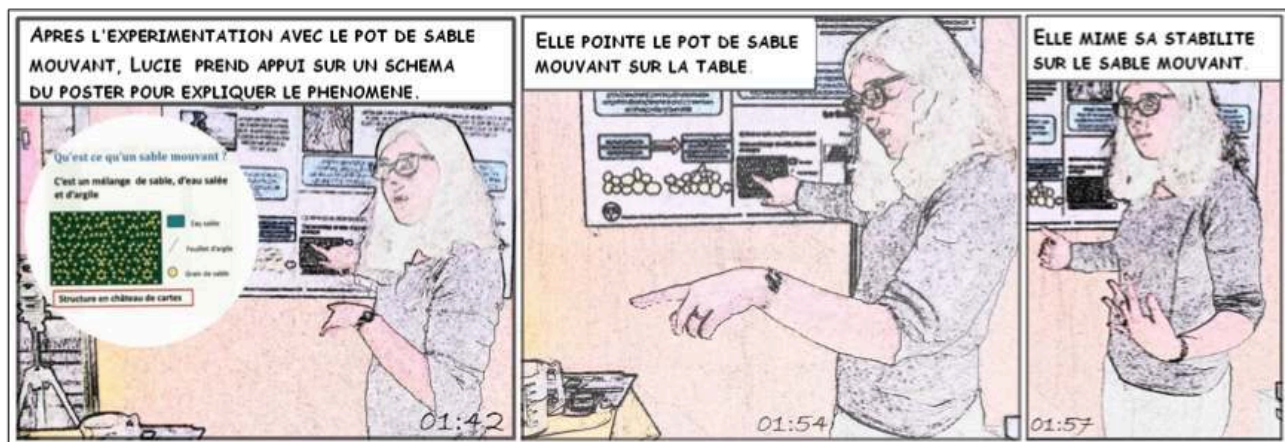

Figure 9 : Annotations réciproques sur une double page (partie descriptive de la situation) Extrait de la thèse (Goujon, 2016, p. 314-315)
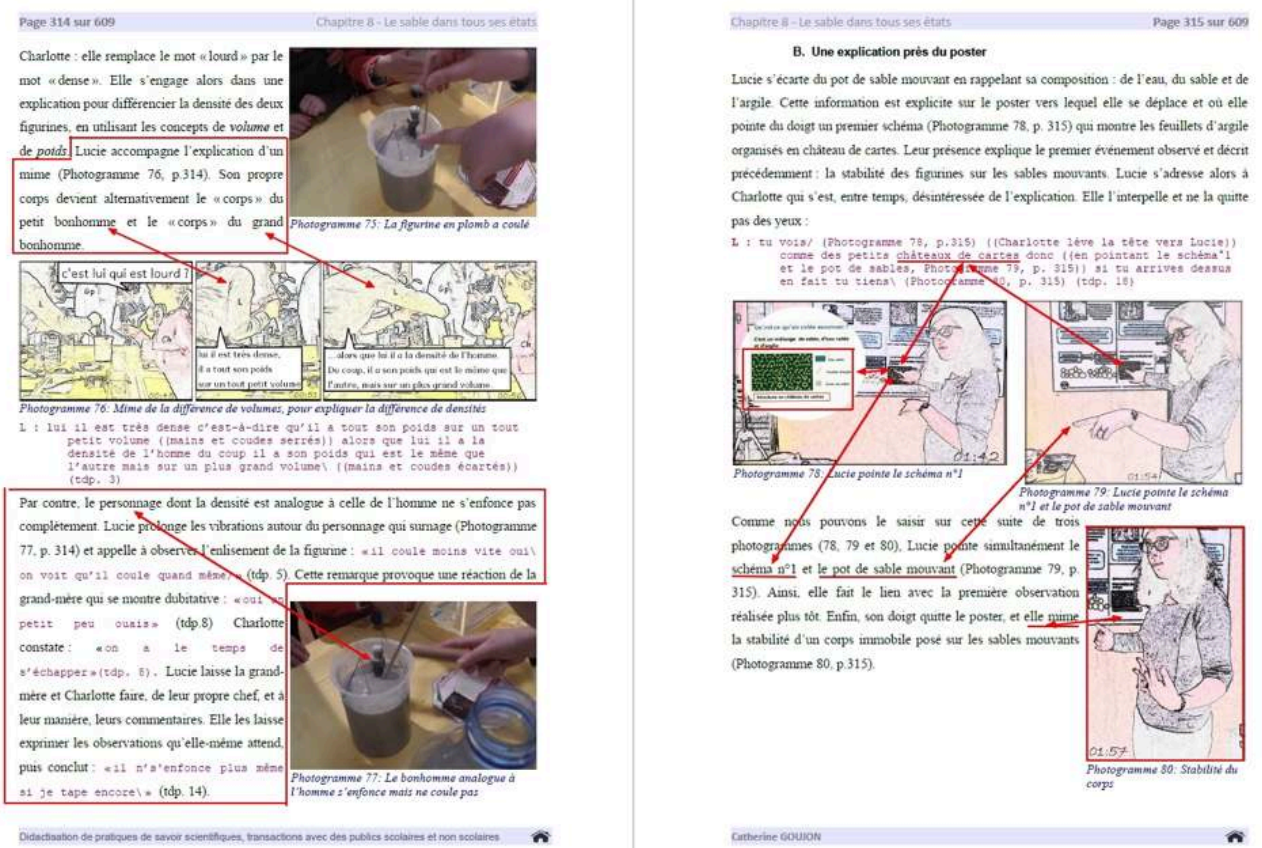

Sur un poster, l'espace intericonique est gommé et complété par des didascalies. Elles donnent des informations succinctes (Figure 8). Elles sont éventuellement complétées par un court texte (Figure 2, pour voir la séquence dans son contexte). Sur ce poster, un $Q R$ code et un lien permettent d'accéder à l'extrait vidéo, objet de l'étude de cas.

Dans une production dévolue à une lecture en solitaire (article, thèse), les images sont associées et juxtaposées aux textes (Figure 9). Elles sont toujours lisibles de façon séquentielle, selon les besoins du lecteur, mais l'espace intericonique est dédié à la description ou à l'analyse de l'auteur, sous la forme d'un texte. Le texte et les séquences d'images s'annotent réciproquement. L'image ne vient pas seulement illustrer le texte. Elle annote le texte, "elle indique au lecteur la manière dont le texte doit être lu " (Sensevy, 2011a, p. 300). Et le texte annote l'image, « il indique au lecteur ce qu'il doit regarder dans l'image" (ibid., p. 301). Ce processus d'énonciation est au cœur de l'ontologie des SHTIS. Il tient à l'unité de production de l'image et du texte par l'auteur. Les SHTIS sont des documents composites homogènes. 


\section{Répétition d'images pour la description, puis l'analyse}

Il n'est pas rare de trouver plusieurs usages des mêmes images dans un même document. Une phase de description des situations, en langage familier des connaisseurs des pratiques étudiées (Sensevy, 2011b), permet de s'acculturer avec la situation. Le chercheur propose ensuite une phase d'analyse de cette même situation, avec des concepts théoriques (pour notre part, issus de la TACD).

\section{Résumé de la phase descriptive (Goujon, 2016, p. 314-315)}

Dans un premier temps, la médiatrice pose deux figurines sur un sable mouvant. Elles ne s'enfoncent pas. Par contre, sous l'effet de l'agitation du sable mouvant, la figurine en plomb coule complètement (Photogramme 75, Figure 9), et l'autre, dont la densité est comparable à celle de l'homme, ne s'enfonce que légèrement (Photogramme 77, Figure 9). L'homme ne peut pas être englouti dans un sable mouvant. Dans un second temps, la médiatrice donne des explications à l'aide de schémas sur un poster (Figure 9, Photogrammes 78, 79 et 80 ). L'argile présente dans le sable mouvant se présente sous la forme de feuillets qui se disposent naturellement en une structure de châteaux de cartes. Ils rigidifient le matériau qui peut soutenir des objets (des humains). Cependant, quand le sable mouvant est agité (par des piétinements, par exemple), les châteaux de cartes s'écroulent et les objets s'enfoncent. Selon que leur densité est plus élevée que celle du sable mouvant (cas du plomb) ou plus basse (cas de celle du corps humain), l'objet est englouti ou flotte.

\section{Résumé de la phase analytique (ibid., p. 326)}

À la page 326 de la thèse (Figure 10), le chercheur analyse comment la médiatrice fait le lien entre les schémas du poster et l'expérimentation réalisée précédemment, mais dont il ne reste que le matériel inerte sur la table.

Le milieu, dans le deuxième temps de la situation, au moment 01:54 (Photogramme 93, Figure 10), est principalement composé du schéma pointé sur le poster et du discours de la médiatrice. Elle donne des explications scientifiques sur la composition du sable mouvant et sur le comportement de l'argile. Le savoir issu de l'expérience réalisée quelques minutes plus tôt a été institué (sable mouvant non agité est solide) et fait partie du contrat entre elle et son public. Ce savoir est un élément qui entre dans un arrière-plan commun, dans le contrat; Matheron et Salin (2002, p. 63) parlent de "mémoire officielle». D’un geste, la médiatrice rappelle l'événement observé lors de la simulation dans le pot de sable mouvant pour le lier à l'explication chimique, et ainsi aider son public à la compréhension du phénomène.

Des annotations graphiques, sous la forme de flèches et de cercles orientent l'attention du lecteur. Ils font du lien entre le modèle analogique de sable mouvant (SM sur le schéma) sur lequel a porté l'expérimentation de la scientifique et les schémas explicatifs symboliques (Photogramme 93, Figure 10).

Pour retirer toute ambiguïté et soutenir son analyse, le chercheur limite les interprétations erronées (la médiatrice pourrait, par exemple, montrer du doigt la petite fille qui se trouve dans le public pour lui signifier qu'elle s'adresse à elle). Le cadrage du photogramme ne permet pas de voir ce qui est pointé par la scientifique. L'auteure rappelle donc l'organisation spatiale du stand avec un plan, sur la même page (Plateau de jeu 13, Figure 10). De la même manière, C. Spottorno et G. Abril (2017 
[2016]) ne se privent pas d'introduire des cartes géographiques personnalisées dans leur album pour situer les événements qu'ils décrivent.

Figure 10 : Annotations graphiques et plan, limitateurs d'interprétations erronées (partie analytique de la situation) - Extrait de la thèse (Goujon, 2016, p. 326)

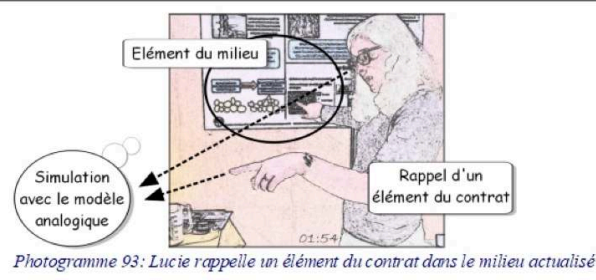

le pot de sable mouvant. Le regard accompagne le geste déictique. Ensemble, regard et pointage matérialisent l'endroit où se situait l'action qu'il faut rappeler à la mémoire. Lucie et Alain prennent en Légende charge le rappel de la situation précédente. Matheron et Salin écrivent que "l'action enseignante reconstruit publiquement la mémoire officielle pour mener à bien le projet d'enseignement " (2002, p. 63).

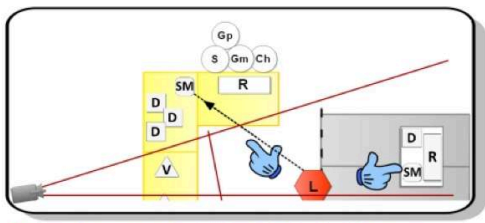
- Transactants

Ch Public (ex. : Charlotte) L Médiatrice (ex. : Lucie) $\{$ Main de la médiatrice - Dispositifs exposés

R $\quad$ Rides de sable

D Dilatance

Plateau_de jeu 13: Reconstitution de la scène didactique, $\overline{\mathrm{SM}} \quad$ Sables mouvants l'espace dans le champ et hors champ de la caméra

Parfois, il n'y a plus de trace tangible de la situation. Le doigt et le regard fixent, et «réifient », l'endroit où est survenu un événement institué plus tôt (Plateau_de_jeu 13, p. 323). Elle donne ainsi à voir un élément symbolique du milieu (le schéma). De l'autre main, appuyée par son regard, elle rappelle l'élément du contrat précédemment élaboré : le grand bonhomme qui s'est enfoncé dans le sable mouvant «jusqu'à la taille ».

\section{Transcription du discours des enquêtés}

Les vignettes comportent généralement peu de texte; la contextualisation de la production du locuteur suffit. Le lecteur a accès au tour de parole complet dans le transcript associé : les paragraphes en retrait sont saisis dans une autre police de caractère (Figure 11). 
Figure 11 : Association du transcript aux photogrammes, avec renvois aux légendes - Extrait de la thèse (Goujon, 2016, p. 315)

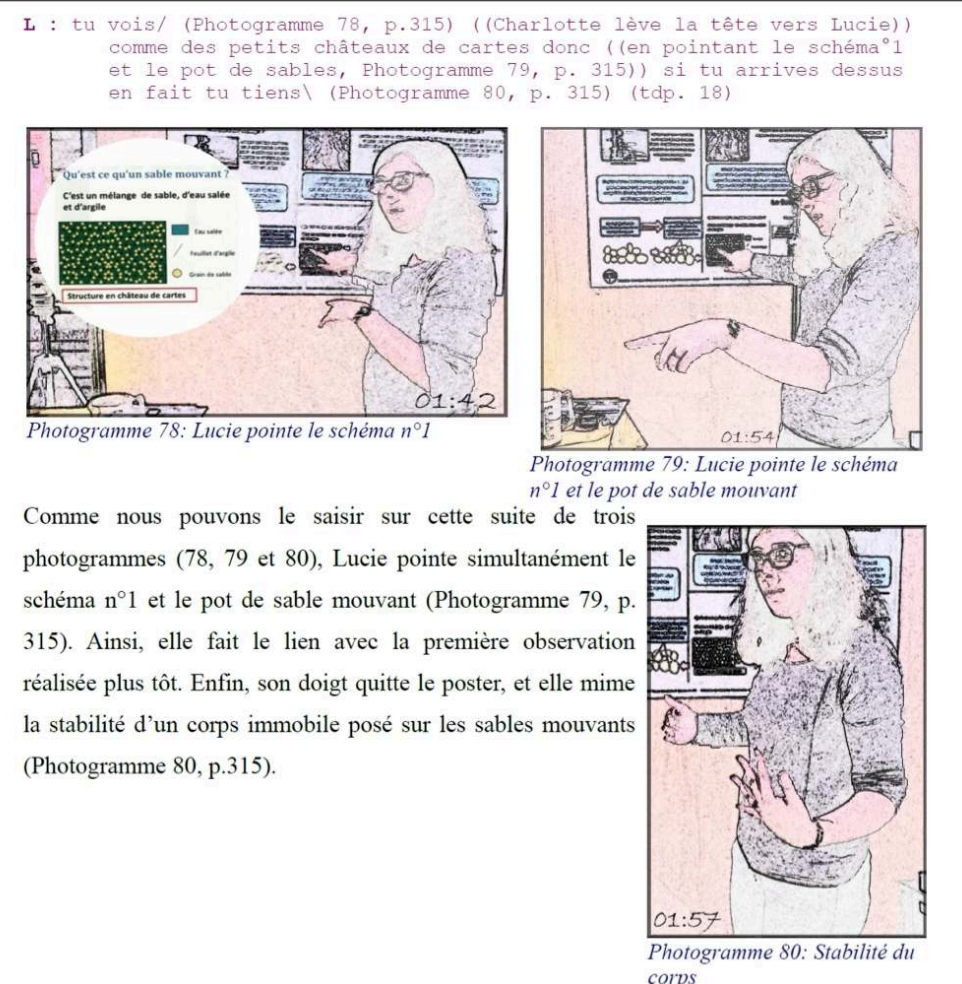

L'introduction de bulles et d'onomatopées vise aussi la possibilité de saisir la production des discours dans l'action. Les paroles prononcées par le personnage que nous représentons sont issues de la transcription des énoncés oraux des acteurs en situation didactique. Inscrire ces discours dans l'image permet au chercheur de contextualiser leur production (Figure 12). Ils sont un écho (souvent plus sommaire) des extraits de transcripts présents dans le corps des descriptions ou des analyses scientifiques. Les inférences que le lecteur opère alors, à partir de la représentation graphique de l'action, enrichit sa seule interprétation du transcript.

Figure 12 : Insertion double des paroles, dans l'image et dans le transcript - Extrait de la thèse (Goujon, 2016, p. 314)

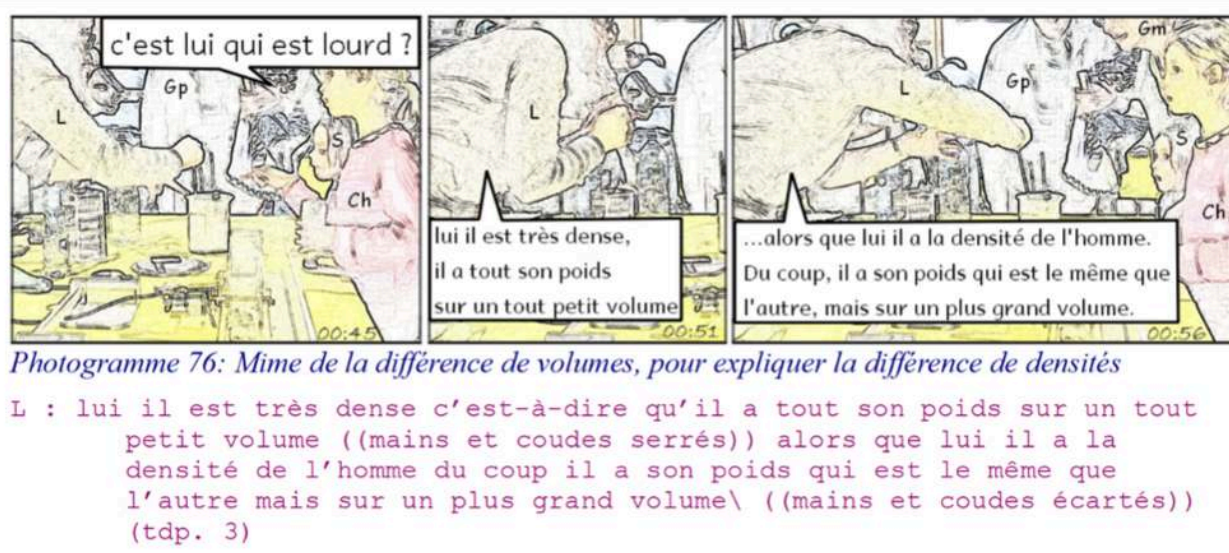




\section{Représentations du temps et des durées}

L'usage des films d'étude permet l'indexation précise des épisodes (frises synoptiques) et des événements (time code). Ces time code sont insérés dans l'image, tant pour faciliter l'analyse du chercheur que pour donner des repères temporels au lecteur. Cependant, leur instantanéité reste toute relative : l'image sélectionnée est emblématique d'une action dont l'empan temporel peut être plus étendu. Ainsi, quelques effets de durées inhérents à l'image fixe peuvent être mis au service des situations étudiées.

\section{Absence de cadre}

Pour certaines actions qui durent plusieurs minutes (et qui pourraient durer encore plus longtemps) la solution peut être la présentation d'une seule image, sans time code ni cadre. Cette absence de limites graphiques renforce l'idée d'une action « intemporelle » même si sa durée peut être quantifiée. Selon W. Eisner (2009 [1985], p. 32), la case est « le critère selon lequel s'évalue l'illusion du temps » dans les bandes dessinées. Ainsi, les multiples prises d'indices et actions nécessaires au réglage d'une maquette d'hydrogéologie sont-elles représentées, symboliquement, sur un seul photogramme sans cadre, et légendé de plusieurs étiquettes (Figure 13).

Figure 13 : Mise en eau de la maquette et stabilisation de la rivière, contrôles et réglages - Extrait de la thèse (Goujon, 2016, p. 177)

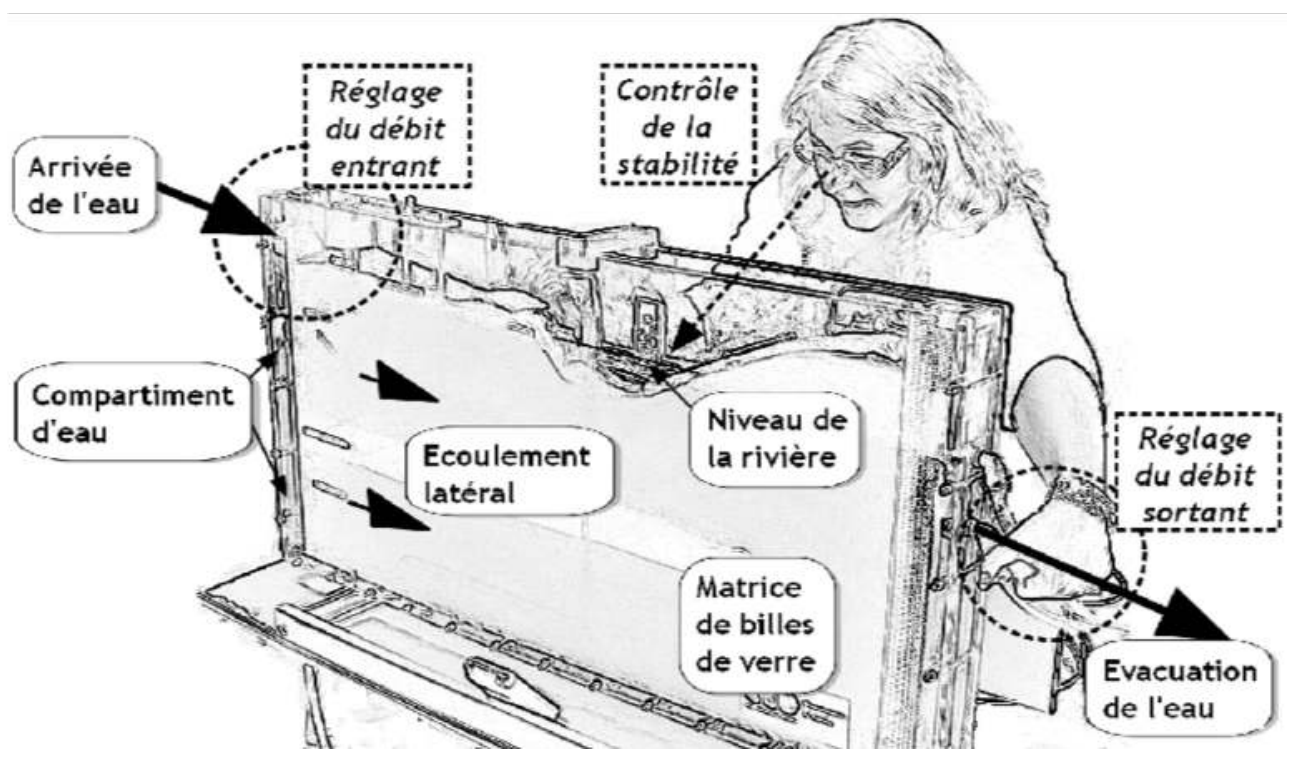

Liéron et Dahan (2019) utilisent une représentation similaire pour suggérer le cumul d'indices de Sherlock Holmes après sa collecte de témoignages. Les vignettes qui entourent le visage concentré de l'enquêteur récapitulent symboliquement les situations qui ont permis leur recueil.

\section{Des bulles qui modifient la durée de l'instantanéité du photogramme}

Avec l'introduction de bulles, le photogramme n'est pas « dépourvu de durée interne " (Groensteen, 2008, p. 25). La durée du discours contenu dans les phylactères déborde sur le moment choisi par l'auteur de l'image. Les dessinateurs de BD en jouent 
constamment: les conversations internes aux vignettes ne sauraient être des instantanés.

(14), le médiateur scientifique d'un stand sur les os et les articulations ne propose, comme milieu, que l'omoplate du squelette. Précédemment, les élèves ont facilement nommé la série d'os et d'articulations montrés. En effet, avant cette visite à la fête de la science, la classe de CE2 a manipulé des radiographies et des documents didactiques, des schémas légendés du squelette. Le médiateur pointe donc, finalement, sur un modèle de squelette en plastique (échelle 1), une omoplate. Il est immobile pendant plusieurs secondes. À son invite d'identifier l'os, les élèves suggèrent des noms. Leurs propositions orales entrent dans le milieu et sont validés ou pas par le médiateur. L'apprentissage est très succinct en ce tout début de visite. Il s'agit ici, pour l'animateur du stand, à ce moment de la rencontre, d'identifier les savoirs antérieurs du groupe d'élèves, le déjà-là présent pour aborder de nouveaux savoirs (les fractures ou les maladies, leur réparation et les comportements qui peuvent réduire leurs risques). Il ajustera son intervention à sa connaissance des savoirs acquis précédemment par le groupe.

Figure 14 : Le temps du discours affecte l'instantanéité du photogramme - Extrait de la thèse (Goujon, 2016, p. 127)

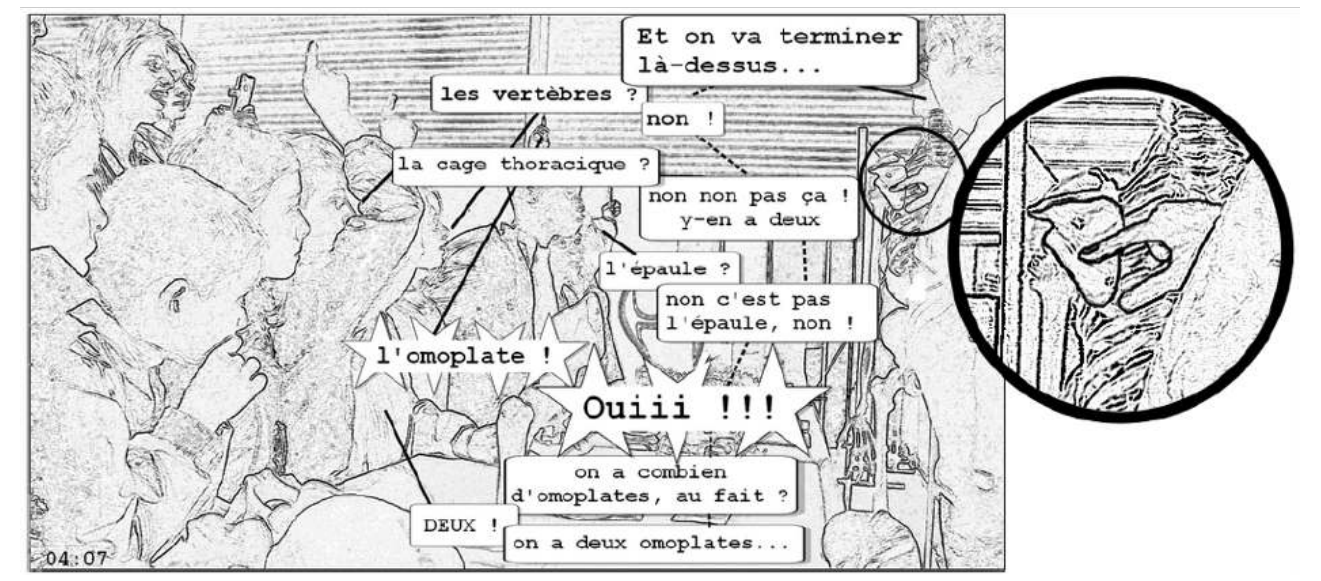

Un milieu composé d'éléments discursifs peut être illustré par un seul photogramme. L'enrichissement du milieu par divers éléments, discursifs et visuels, nécessite par contre une séquence de photogrammes (Figure 8). Ils sont quelquefois très rapprochés dans le temps (Figure 15).

Plus tard, le même médiateur montre aux élèves la relation entre les fractures et la forme des os. Il attire l'attention de la classe sur la fragilité du col du fémur due à son étroitesse.

La séquence d'images illustrant la gestuelle du médiateur, associée au transcript, montre la densité du milieu que le médiateur propose aux élèves: le nombre d'éléments visuels et discursifs à mettre en relation est important. 
Figure 15 : Densité d'éléments de savoir sur une courte durée - Extrait du mémoire (Goujon, 2013, p. 46)

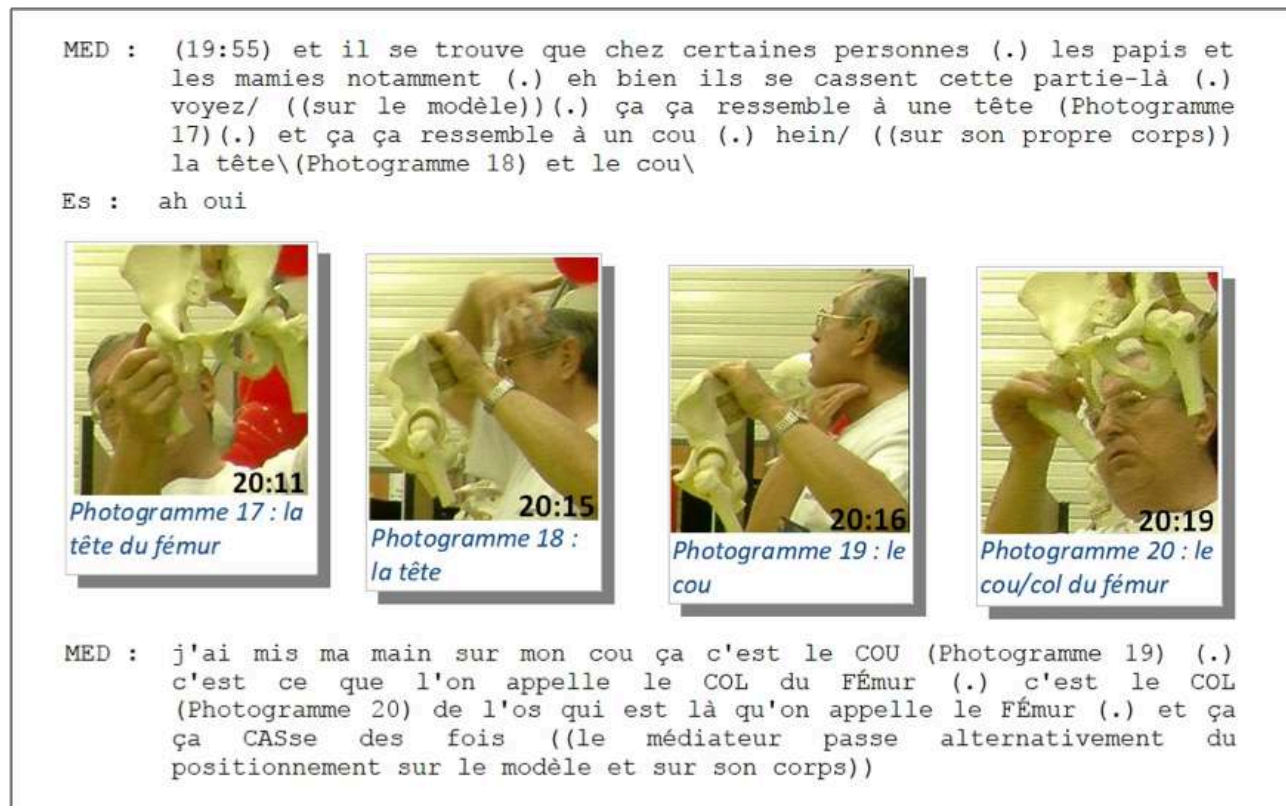

Des épisodes de durées identiques peuvent donc être traduits, selon la densité et la forme des éléments du milieu, par une seule vignette (Figure 14) ou par une séquence d'images (Figure 15). Ils font l'objet de descriptions et d'analyses différentes dans nos productions.

\section{Conclusion}

Les documents composites que nous proposons, les SHTIS, présentent une homogénéité qui facilite leur lecture. Textes et images, produits de façon synchrone, sont organiquement liés. Le chercheur est auteur des différents éléments du document composite. Il tresse ses photogrammes et son texte. Il y développe son voir-comme, sa description, qui accompagne le regard du lecteur, et son analyse didactique.

Le chercheur fonde ses analyses sur des études de cas. Les situations, restituées par l'image, sont, pour lui, dans la genèse de son travail, une donnée première. Il l'offre au lecteur, non seulement comme une illustration de ses propos, mais comme un élément constitutif de son travail. La dépiction des situations et des actions, au-delà de leur description, enrichit le texte d'innombrables informations à identifier comme des compléments, pour qui voudrait les lire et les analyser indépendamment du texte.

Les éléments iconiques sont conçus par le même auteur. L'unité (1) du traitement des photogrammes et (2) de la syntaxe des annotations graphiques facilite la lecture des séquences d'images, après un temps d'appropriation, comme dans toute œuvre graphique.

Les images entre elles, ainsi que les images et le texte, s'annotent réciproquement. Cette mise en systèmes de textes et d'images, voire d'enregistrements audio ou vidéo, relève d'une forme de rhétorique de nos productions scientifiques. Certains procédés empruntés à la bande dessinée ou aux BD de reportage sont combinés aux exigences des textes scientifiques. Ainsi le caractère elliptique de la BD est en grande partie évité. 


\section{BIBLIOGRAPHIE}

BENTHAM, J. (2002) [1791]. Panoptique. Mémoire sur un nouveau principe pour construire des maisons d'inspection, et nommément des maisons de force. Trad. de l'anglais par É. Dumont. Paris : Éd. Mille et une nuits.

BESNIER, S. (2016). Le travail documentaire des professeurs à l'épreuve des ressources technologiques. Le cas de l'enseignement du nombre à l'école maternelle. Thèse en science de l'éducation : Université de Bretagne Occidentale.

BLOCHER, J.-N. (2018). Comprendre et montrer la transmission du savoir : Les systèmes hybrides texteimage-son comme lieux de production et d'écriture des phénomènes. Une illustration en théorie de l'action conjointe en didactique. Thèse de doctorat en didactique : Université de Bretagne Occidentale.

Collectif Didactique pour enseigner (2019). Didactique pour enseigner. Rennes : Presses universitaires de Rennes.

EISNER, W. (2009) [1985]. Les clés de la bande dessinée. 1, L'art séquentiel. Trad. l'anglais par A. Clare. Paris : Delcourt.

EISNER, W. (2010) [1996]. Les clés de la bande dessinée. 2, La narration. Trad. l'anglais par A. Capuron. Paris : Delcourt.

FOREST, D. (2006). Analyse proxémique d'interactions didactiques. Thèse de doctorat en sciences de l'homme et de la société : Université de Rennes 2.

GOODMAN, N. (2011) [1969]. Langages de l'art. Une approche de la théorie des symboles. Trad. de l'anglais (États-Unis) par J. Morizot. Paris : Librairie Arthème Fayard.

GOSCINNY, R., \& SEMPÉ, J.-J. (1961). Les Récrés du petit Nicolas. Paris : Denoël.

GOUJON, C. (2013). Action des professeurs dans un travail conjoint entre un médiateur scientifique, une professeure des écoles et ses élèves de CE2. Sortie scolaire au Festival des sciences. Visite d'un stand Os et articulations. Mémoire de master en éducation, apprentissage \& didactique : Université de Rennes 2.

GOUJON, C. (2016). Didactisation de pratiques de savoir scientifiques, transactions avec des publics scolaires et non scolaires. Des scientifiques de leur laboratoire à la Fête de la science. Thèse en sciences de l'éducation : Université de Bretagne Loire. En ligne : https://hal.archives-ouvertes.fr/ tel-01692314.

GOUJON, C. (2018). « Enjeux de systèmes hybrides texte-image-son dans une recherche en didactique des sciences ». Weekend Jeunes Chercheurs de l'ARDiST. 25-26 mars 2018 [Poster]. Saint-Malo. En ligne : https://wejch-ardist-stmalo2018.log.bzh/2018/02/23/enjeux-de-systemeshybrides-texte-image-son-dans-une-recherche-en-didactique-des-sciences/.

Goujon, C. (2019). « Avalanche... Ou comment on apprend à lire une représentation graphique. Une visite guidée ». In : Collectif Didactique pour enseigner. Didactique pour enseigner. Rennes : Presses universitaires de Rennes, p. 445-464.

GROENSTEEN, T. (2008). La bande dessinée. Mode d'emploi. Bruxelles : Éd. Les Impressions Nouvelles. GUIBERT, E., LEFÈVRE, D., \& LEMERCIER, F. (2010) [2003]. Le photographe. Paris : Dupuis.

HALL, E. T. (1971) [1966]. La dimension cachée. Trad. de l'anglais (États-Unis) par A. Petita, Paris : Éditions du Seuil. 
INGOLD, T. (2017). « Les matériaux de la vie ». Trad. de l'anglais par C. Paris. Socio-anthropologie 35, p. 23-43. En ligne : https://doi.org/10.4000/socio-anthropologie.2519.

LEPAGE, E., \& LEPAGE, F. (2014). La Lune est blanche. Paris : Futuropolis.

LIÉRON, C., \& DAHAN, B. (2019). Dans la tête de Sherlock Holmes. 1, L'affaire du ticket scandaleux.

Roubaix : Ankama.

MATHERON, Y., \& SALIN, M.-H. (2002). « Les pratiques ostensives comme travail de construction d'une mémoire officielle de la classe dans l'action enseignante ». Revue française de pédagogie, 141, p. 57-66. En ligne : http://ife.ens-lyon.fr/publications/edition-electronique/revue-francaise-depedagogie/INRP_RF141_6.pdf.

Mccloud, S. (2007) [1999]. L'art invisible. Trad. de l'anglais par D. Petitfaux. Paris : Delcourt.

MUYBRIDGE, E. (1878). The horse in motion. En ligne : https://commons.wikimedia.org/wiki/

File:The_Horse_in_Motion.jpg.

MORALES IBARRA, G. S. Z. (2014). L'enseignement et l'apprentissage de la représentation. Une étude de cas en maternelle : le «jeu des trésors ». Thèse en sciences de l'éducation : Université de Bretagne Occidentale. En ligne : https://tel.archives-ouvertes.fr/tel-01366889.

ROESSLÉ, S. (2009). Construction de dispositions didactiques chez le jeune enfant : des pratiques éducatives familiales - bébés nageurs et jeu à la maison - vers l'entrée dans les apprentissages scolaires à l'école maternelle. Thèse de doctorat. Université de Rennes 2. En ligne : https://hal.archives-ouvertes.fr/ tel-00472545.

SENSEVY, G. (1999). Éléments pour une anthropologie de l'action didactique. Université de Provence AixMarseille : Marseille.

SENSEVY, G. (2011a). «Comprendre l'action didactique : Méthode et jeux d'échelle ». In : Sensevy, G., Le sens du savoir. Éléments pour une théorie de l'action conjointe en didactique. Bruxelles : De Boeck, p. 217-301. En ligne : https://www.cairn.info/le-sens-du-savoir--9782804163457-page-181.htm.

SENSEVY, G. (2011b). Le sens du savoir. Éléments pour une théorie de l'action conjointe en didactique. Bruxelles : De Boeck. En ligne : https://www.cairn.info/le-sens-du-savoir--9782804163457page-181.htm.

SENSEVY, G. (2013). « Filmer la pratique : Un point de vue de la théorie de l'action conjointe en didactique ». In : Veillard L. \& Tiberghien A. (dirs), ViSA Instrumentation de la recherche en éducation. Paris : Éd. de la Maison des sciences de l'homme.

SPOTTORNo, C., \& ABRIL, G. (2017) [2016]. La fissure. Trad. de l'espagnol par F. Fiore. Paris : Gallimard Bande dessinée.

WINKIN, Y. (2001) [1996]. Anthropologie de la communication. De la théorie au terrain. Paris : De Boeck/ Éditions du Seuil.

\section{NOTES}

1. Le concept de «milieu didactique » sera réduit à celui de « milieu » dans la suite du texte.

2. Les stands étaient respectivement proposés et animés par l'Institut de Physique de Rennes (IPR), l'Institut National de la Santé Et de la Recherche Médicale (INSERM) et 
l'Observatoire des Sciences de l'Univers de Rennes (OSUR) associé au Bureau de Recherche Géologique Minière (BRGM) de Bretagne.

3. Expression attribuée en 1977 au dessinateur néerlandais J. Swarte pour décrire le graphisme d'Hergé.

4. Cet effet est disponible avec le logiciel VLC par exemple.

5. Leur lisibilité dépend du média qui les diffuse. La nature numérique de cet article permet l'agrandissement des images, le cas échéant.

6. Élèves et professeurs sont considérés ici comme les personnes qui apprennent et celles qui enseignent (Collectif Didactique pour Enseigner, 2019, p. 602).

\section{RÉSUMÉS}

Les textes scientifiques en didactique présentent de plus en plus souvent des documents composites qui intègrent des séquences d'images telles des bandes dessinées (BD). Nous abordons ici un corpus de textes en didactique des sciences. Que montrent ces compositions et comment sont-elles construites? Quelles relations les images entretiennent-elles avec le texte ? Ce type de document composite a la particularité d'être homogène : texte et images sont construits par le même auteur et s'annotent mutuellement. Ils constituent des systèmes hybrides texte - image son (SHTIS), outils du chercheur-auteur qui donne ainsi à comprendre et à voir, à son lecteur, les situations didactiques qu'il étudie. Dans cet article, nous nous référons à la théorie de l'action conjointe en didactique (TACD) ainsi qu'aux recherches de théoriciens de la BD.

Scientific texts in didactics increasingly present composite documents that incorporate image sequences such as comic strips. We discuss here a corpus of texts in science didactics. What do these compositions show and how are they constructed? How do the images relate to the text? This type of composite document has the particularity of being homogeneous: text and images are built by the same author and annotate each other. They constitute Picture-Text-Audio Hybrid Systems (PTAHS), tools of the researcher-author who thus gives to understand and see, to his reader, the didactic situations he studies. In this article, we refer to the joint action theory in didactics (JATD) as well as to the research of comic book theorists.

\section{INDEX}

Mots-clés : bande dessinée, BD, SHTIS, systèmes hybrides texte - image - son, TACD, théorie de l'action conjointe en didactique

Keywords : comics, JATD, joint action theory in didactics, Picture-Text-Audio Hybrid Systems, PTAHS 
AUTEUR

\section{CATHERINE GOUJON}

Université de Bretagne Occidentale/INSPE de Bretagne, Cread, F-35043 Rennes, France 